\title{
A New Lagrangian Asymptotic Solution for Gravity-Capillary Waves in Water of Finite Depth
}

\author{
Hung-Chu Hsu, Chiu-On Ng and Hwung-Hweng Hwung \\ Communicated by A. Constantin
}

\begin{abstract}
A third-order Lagrangian asymptotic solution is derived for gravity-capillary waves in water of finite depth. The explicit parametric solution gives the trajectory of a water particle and the wave kinematics for Lagrangian points above the mean water level, and in a water column. The water particle orbits and mass transport velocity as functions of the surface tension are obtained. Some remarkable trajectories may contain one or multiple sub-loops for steep waves and large surface tension. Overall, an increase in surface tension tends to increase the motions of surface particles including the relative horizontal distance travelled by a particle as well as the time-averaged drift velocity.
\end{abstract}

Mathematics Subject Classification (2010). Primary 76B15; Secondary 35Q35, 34C05, 34C25.

Keywords. Gravity-capillary waves, Lagrangian, particle trajectory, surface tension.

\section{Introduction}

Although much attention has been paid to Lagrangian water waves in recent years, there exist rather few Lagrangian studies which are specifically for gravity-capillary waves. Gravity-capillary waves are of considerable practical interest not only because of their relevance to the energy transfer between the atmosphere and the ocean, but also because of their contribution to the remote sensing of the ocean surface by airborne and spaceborne radars, and also to the underwater acoustical background generated during the process of bubble formation [33]. In-depth knowledge of the characteristics of gravity-capillary waves is therefore important.

The study of nonlinear gravity-capillary waves was pioneered by Wilton [40]. It was followed by Crapper [14], who studied pure capillary waves in water of infinite depth, and Kinnersley [28], who generalized the theory to finite depth. For nonlinear water waves, the existence of the fully nonlinear equations was proven in $[12,13,38,41]$, firstly for gravity water waves and then for waves incorporating surface tension. Further properties of the flow for these waves were enunciated in $[4,7,8,17,21]$, such as the symmetry of the flow. Recent analytically rigorous results which provide a qualitative description of the particle trajectories for a wide variety of both linear and nonlinear flow are [3-11,15-21,26,27,39]. These works prove that there does not exist any closed particle paths throughout the fluid domain for the irrotational flows detailed therein. The particle motion predicted in these papers is borne out by experimental evidence [2]. Longuet-Higgins [31] and Hogan [23,24] used the Euler-Lagrange transformation to describe particle orbits in capillary waves and nonlinear gravity-capillary waves. Their models are, however, applicable only to particles on the water surface, and cannot be generalized to interior fluid particles.

Recently, some exact solutions using the phase-plane method for particle trajectories in linearized capillary and capillary-gravity water waves have been contributed by Henry $[18,19]$. However, the solutions and the details about the flow under the nonlinear waves are still not available. This paper aims to study particle trajectories of nonlinear gravity-capillary waves completely based on the Lagrangian system, and to derive asymptotic solutions that can be used to describe the dynamics for the entire flow field. Previous works on progressive, standing and short-crested gravity waves have been summarized in the 
papers by Chen and Hsu [1] and Hsu et al. [25]. In this paper, we look into the effect of surface tension (capillary) on a gravity water wave, the motion of which is assumed to be inviscid, incompressible and irrotational. We will construct asymptotic expansions of the solution in powers of the wave amplitude, which is assumed to be small. Approximate solutions are derived up to the third order, without admitting internal resonances [35]. A detailed analysis of influences of the surface tension is then carried out.

The problem formulation, and the procedures for constructing asymptotic solutions are described in Sect. 2. In Sect. 3, we derive equations for the properties of surface-particle trajectories, and present results for some selected gravity-capillary waves. In Sect. 4 the trajectories of surface and subsurface particles, and wave profiles of nonlinear capillary-gravity waves are presented. Finally, some concluding remarks are given in Sect. 5 .

\section{Formulation of the Problem}

We consider steady, symmetric, periodic, nonlinear gravity-capillary waves propagating on the surface of an incompressible and inviscid body of water of uniform depth. The fluid motion is taken to be twodimensional and irrotational, and the wave is right-going. We choose Cartesian axes with $x$ pointing horizontally to the right and $y$ vertically downward. The mathematical problem is formulated in terms of Lagrangian variables, $a$ and $b$, which define the original position of individual fluid particles. At any time $t$, we let $b=0$ be the free surface, and $b=d$ be the bottom. The Cartesian coordinates $(x(a, b, t), y(a, b, t))$ of fluid particles and the fluid pressure $P(a, b, t)$ are the unknowns. Based on the Lagrangian description, the governing equations and boundary conditions for two-dimensional irrotational free-surface flow are summarized as follows:

$$
\begin{gathered}
J=\frac{\partial(x, y)}{\partial(a, b)}=1 \\
x_{a t} y_{b}-x_{b t} y_{a}+x_{a} y_{b t}-x_{b} y_{a t}=\frac{\partial\left(x_{t}, y\right)}{\partial(a, b)}+\frac{\partial\left(x, y_{t}\right)}{\partial(a, b)}=0 \\
x_{a t} x_{b}-x_{b t} x_{a}+y_{a t} y_{b}-y_{b t} y_{a}=\frac{\partial\left(x_{t}, x\right)}{\partial(a, b)}+\frac{\partial\left(y_{t}, y\right)}{\partial(a, b)}=0 \\
\frac{\partial \phi}{\partial a}=x_{t} x_{a}+y_{t} y_{a}, \quad \frac{\partial \phi}{\partial b}=x_{t} x_{b}+y_{t} y_{b}, \\
\frac{p}{\rho}=-\frac{\partial \phi}{\partial t}-g y+\frac{1}{2}\left(x_{t}^{2}+y_{t}^{2}\right)+\frac{T}{\rho} S \\
S=\left[\frac{\partial^{2} y}{\partial a^{2}} \frac{\partial x}{\partial a}-\frac{\partial^{2} x}{\partial a^{2}} \frac{\partial y}{\partial a}\right] \cdot\left[\left(\frac{\partial x}{\partial a}\right)^{2}+\left(\frac{\partial y}{\partial a}\right)^{2}\right]^{-3 / 2} \\
p=0, \quad b=0 \\
v=y_{t}=0, \quad y=b=-d,
\end{gathered}
$$

In Eqs. (2.1)-(2.7), subscripts $a, b$, and $t$ denote partial derivatives with respect to the specified variables, $T$ is the surface tension coefficient, $S$ is the radius of curvature, $g$ is the gravitational acceleration, $p(a, b, t)$ is water pressure, $\phi(a, b, t)$ is a velocity potential function in the Lagrangian system. Except for Eqs. (2.4) and (2.5), the fundamental physical relationships defining the equations above have been derived previously $[29,34,37,41]$. Equation (2.1) is the continuity equation based on the invariant condition on the volume of a Lagrangian particle; Eq. (2.2) is the differentiation of Eq. (2.1) with respect to time. Equations (2.3) and (2.4) denote the irrotational flow condition and the corresponding Lagrangian velocity potential, respectively. Equation (2.5) is the Bernoulli equation with surface tension for the irrotational flow in the Lagrangian description. The wave motion has to satisfy a number of boundary conditions at the bottom and on the free water surface. On a rigid and impermeable bottom the no-flux bottom boundary condition gives Eq. (2.6). Equation (2.7) is the dynamic boundary condition of zero pressure at the free surface. 


\section{Asymptotic Solutions}

To solve the nonlinear equations (2.1)-(2.7), we introduce the Lagrangian angular frequency $\sigma$ of particle motion which is a function of a nonlinear parameter and the Lagrangian level label $(b)$, making the wave periodic in time $t$ and space $x$ (or $a$ ) in order to avoid a secular term. We use the Lindstedt-Poincare technique that yields uniform expansions to uncover the solutions in the Lagrangian system. In the Lagrangian approach, the particle positions $x$ and $y$, the potential function $\phi$ and pressure $P$ are considered as functions of independent variables $a, b$ and time $t$. Following Chen and Hsu [1], Pierson [37], and Piedra-Cueva [36], these solutions are sought in perturbation series by introducing an ordering parameter, which is inserted to identify the order of the associated term:

$$
\begin{aligned}
& x=x(a, b, t)=a+\sum_{n=1}^{\infty} \epsilon^{n}\left[f_{n}(a, b, \sigma t)+f_{n}^{\prime}\left(b, \sigma_{0} t\right)\right], \\
& y=y(a, b, t)=b+\sum_{n=1}^{\infty} \epsilon^{n}\left[g_{n}(a, b, \sigma t)+g_{n}^{\prime}\left(b, \sigma_{0} t\right)\right], \\
& \phi=\phi(a, b, t)=\sum_{n=1}^{\infty} \epsilon^{n}\left[\phi_{n}(a, b, \sigma t)+\phi_{n}^{\prime}\left(b, \sigma_{0} t\right)\right], \\
& p=p(a, b, t)=-\rho g b+\sum_{n=1}^{\infty} \epsilon^{n} p_{n}(a, b, \sigma t), \\
& \sigma=\sigma(b)=\sigma_{0}+\sum_{n=1}^{\infty} \epsilon^{n} \sigma_{n}(b)=2 \pi / T_{L}(b),
\end{aligned}
$$

where the Lagrangian variables $(a, b)$ are defined as the two characteristic parameters. In these expressions, $f_{n}, g_{n}, \phi_{n}$ and $p_{n}$ are expected to be associated with the nth-order harmonic solutions. $f_{n}^{\prime}, g_{n}^{\prime}$ and $\phi_{n}^{\prime}$ are non-periodic functions that increase linearly with time. $\sigma=2 \pi / T_{L}$ is the angular frequency of particle motion or the Lagrangian angular frequency for a particle reappearing at the same elevation. $T_{L}$ is the corresponding period of particle motion. Upon substituting Eqs. (3.1)-(3.5) into Eqs. (2.1)-(2.7), and collecting terms of equal order, we obtain a sequence of nonhomogeneous governing equations that can be solved successively, as shown in the following sections. Recently, it was proven that the convergence and consequent validity of the power series expansions from a mathematical rigorous viewpoint is ensured since the streamlines are analytic $[8,22]$.

\subsection{First-Order Approximation}

Collecting terms of order $\epsilon$, the governing equations and the boundary conditions can be obtained as follows:

$$
\left\{\begin{array}{l}
f_{1 a}+f_{1 a}^{\prime}+g_{1 b}+g_{1 b}^{\prime}=0 \\
\sigma_{0}\left(f_{1 a \sigma t}+f_{1 a \sigma_{0} t}^{\prime}+g_{1 b \sigma t}+g_{1 b \sigma_{0} t}^{\prime}\right)=0 \\
\sigma_{0}\left(f_{1 b \sigma t}+f_{1 b \sigma_{0} t}^{\prime}-g_{1 a \sigma t}-g_{1 a \sigma_{0} t}^{\prime}\right)=0 \\
\phi_{1 a}+\phi_{1 a}^{\prime}=\sigma_{0}\left(f_{1 \sigma t}+f_{1 \sigma_{0} t}^{\prime}\right) \\
\phi_{1 b}+\phi_{1 b}^{\prime}=\sigma_{0}\left(g_{1 \sigma t}+g_{1 \sigma_{0} t}^{\prime}\right) \\
\frac{p_{1}}{\rho}=-\sigma_{0}\left(\phi_{1 \sigma t}+\phi_{1 \sigma_{0} t}^{\prime}\right)-g\left(g_{1}+g_{1}^{\prime}\right)+\frac{T}{\rho}\left\{\left(g_{1 a a}+g_{1 a a}^{\prime}\right)+\left[\sigma_{0 a}\left(g_{1 \sigma t}+g_{1 \sigma_{0} t}^{\prime}\right)\right]_{a}\right\} \\
p_{1}=0, \quad b=0, \\
g_{1 \sigma t}=g_{1 \sigma_{0} t}^{\prime}=0, \quad b=-d
\end{array}\right.
$$


The flow is assumed periodic with a crest at $a=0$ and $t=0$, and hence the first-order solution can be easily written as

$$
\begin{gathered}
f_{1}^{\prime}=g_{1}^{\prime}=\phi_{1}^{\prime}=\sigma_{0 a}=\sigma_{0 b}=0, \\
f_{1}=-\alpha \frac{\cosh k(b+d)}{\cosh k d} \sin (k a-\sigma t), \\
g_{1}=\alpha \frac{\sinh k(b+d)}{\cosh k d} \cos (k a-\sigma t), \\
\phi_{1}=\frac{\alpha \sigma_{0}}{k} \frac{\cosh k(b+d)}{\cosh k d} \sin (k a-\sigma t), \\
\frac{p_{1}}{\rho}=-\alpha \rho g\left(1+\frac{T k^{2}}{\rho g}\right) \frac{\sinh k b}{\cosh ^{2} k d} \cos (k a-\sigma t), \\
\sigma_{0}^{2}=g k\left(1+\frac{T k^{2}}{\rho g}\right) \tanh k d,
\end{gathered}
$$

where the parameter $\alpha$ represents the amplitude function of the particle displacement; the wave amplitude is as usual taken as $a_{0}=\alpha \tanh k d . \phi_{1}(a, b, t)$ is the first-order Lagrangian velocity potential and $p_{1}(a, b, t)$ is the first-order wave dynamic pressure in the Lagrangian form with pressure $p_{1}=0$ at the free surface $b=0$. Equations (3.7)-(3.11) satisfy all the hydrodynamic equations formulated in Lagrangian terms including the irrotational condition, and differ from Gerstner's wave in infinite water depth that possesses finite vorticity. The dispersion relation shows that the first-order Lagrangian wave frequency $\left(\sigma_{0}\right)$ is the same as that of the first-order Stokes wave frequency in the Eulerian approach [35]. The first-order free surface in Lagrangian coordinates is given by setting $b=0$ in Eqs. (3.8) and (3.9), and is similar to expressions for the profile found from the first-order Eulerian equations.

\subsection{Second-Order Approximation}

Collecting terms of order $\epsilon^{2}$ and using Eq. (3.7), the governing equations and the boundary conditions can be obtained as

$$
\begin{aligned}
& f_{2 a}+f_{2 a}^{\prime}+g_{2 b}+g_{2 b}^{\prime}+f_{1 a} g_{1 b}-f_{1 b} g_{1 a}+\left(\sigma_{1 a} f_{1 \sigma t}+\sigma_{1 b} g_{1 \sigma t}\right) t=0 \\
& \sigma_{0}\left(f_{2 a \sigma t}+f_{2 a \sigma_{0} t}^{\prime}+g_{2 b \sigma t}+g_{2 b \sigma_{0} t}^{\prime}\right)+\sigma_{1}\left(f_{1 a}+g_{1 b}\right)_{\sigma t} \\
& \quad+\sigma_{0}\left(f_{1 a} g_{1 b}-f_{1 b} g_{1 a}\right)_{\sigma t}+\sigma_{1 a} f_{1 \sigma t}+\sigma_{1 b} g_{1 \sigma t} \\
& \quad+\sigma_{0}\left[\sigma_{1 a} f_{1(\sigma t)^{2}}+\sigma_{1 b} g_{1(\sigma t)^{2}}\right] t=0 \\
& \sigma_{0}\left(f_{2 b \sigma t}+f^{\prime}{ }_{2 b \sigma_{0} t}-g_{2 a \sigma t}-g_{2 a \sigma_{0} t}^{\prime}\right)+\sigma_{1}\left(f_{1 b}-g_{1 a}\right)_{\sigma t} \\
& \quad+\sigma_{1 b} f_{1 \sigma t}-\sigma_{1 a} g_{1 \sigma t}+\sigma_{0}\left(f_{1 a} f_{1 b \sigma t}-f_{1 a \sigma t} f_{1 b}+g_{1 a} g_{1 b \sigma t}\right. \\
& \left.\quad-g_{1 a \sigma t} g_{1 b}\right)+\sigma_{0}\left[\sigma_{1 b} f_{1(\sigma t)^{2}}-\sigma_{1 a} g_{1(\sigma t)^{2}}\right] t=0 \\
& \phi_{2 a}+\phi_{2 a}^{\prime}=\sigma_{0}\left(f_{2 \sigma t}+f_{2 \sigma_{0} t}^{\prime}\right)+\sigma_{1} f_{1 \sigma t}+\sigma_{0}\left(f_{1 a} f_{1 \sigma t}+g_{1 a} g_{1 \sigma t}\right)-\sigma_{1 a} \phi_{1 \sigma t} t \\
& \phi_{2 b}+\phi_{2 b}^{\prime}=\sigma_{0}\left(g_{2 \sigma t}+g_{2 \sigma_{0} t}^{\prime}\right)+\sigma_{1} g_{1 \sigma t}+\sigma_{0}\left(f_{1 b} f_{1 \sigma t}+g_{1 b} g_{1 \sigma t}\right)-\sigma_{1 b} \phi_{1 \sigma t} t \\
& \frac{P_{2}}{\rho}=-\left[\sigma_{0}\left(\phi_{2 \sigma t}+\phi_{2 \sigma_{0} t}^{\prime}\right)+g\left(g_{2}+g_{2}^{\prime}\right)\right]-\sigma_{1} \phi_{1 \sigma t} \\
& \quad+\frac{1}{2} \sigma_{0}^{2}\left(f_{1 \sigma t}^{2}+g_{1 \sigma t}^{2}\right)+\frac{T}{\rho}\left\{\left(g_{2 a a}+g_{2 a a}^{\prime}\right)+\left(\sigma_{1 a} t g_{1 \sigma t}\right)_{a}\right. \\
& \left.\quad-2\left(f_{1 a}+f_{1 a}^{\prime}\right)\left(g_{1 a a}+g_{1 a a}^{\prime}\right)-\left(f_{1 a a}+f_{1 a a}^{\prime}\right)\left(g_{1 a}+g_{1 a}^{\prime}\right)\right\}
\end{aligned}
$$


Substituting Eqs. (3.8)-(3.12) into Eqs. (3.13)-(3.15), the second-order governing equations in terms of $\epsilon^{2}$, including the continuity equation and the irrotational condition, are given by

$$
\begin{aligned}
& f_{2 a}+f_{2 a}^{\prime}+g_{2 b}+g_{2 b}^{\prime}=\frac{1}{2} \alpha^{2} k^{2}\left[\frac{\cosh 2 k(b+d)}{\cosh ^{2} k d}+\frac{\cos 2(k a-\sigma t)}{\cosh ^{2} k d}\right] \\
& -\alpha \sigma_{1 b} \frac{\sinh k(b+d)}{\cosh k d} \sin (k a-\sigma t) \cdot t, \\
& \sigma_{0}\left(f_{2 a \sigma t}+f_{2 a \sigma_{0} t}^{\prime}+g_{2 b \sigma t}+g_{2 b \sigma_{0} t}^{\prime}\right)=\alpha^{2} k^{2} \sigma_{0} \frac{\sin 2(k a-\sigma t)}{\cosh ^{2} k d} \\
& \quad-\alpha \sigma_{1 b} \frac{\sinh k(b+d)}{\cosh k d} \sin (k a-\sigma t)+\alpha \sigma_{0} \sigma_{1 b} \frac{\sinh k(b+d)}{\cosh k d} \cos (k a-\sigma t) \cdot t, \\
& \sigma_{0}\left(f_{2 b \sigma t}+f_{2 b \sigma_{0} t}^{\prime}-g_{2 a \sigma t}-g_{2 a \sigma_{0} t}^{\prime}\right)=\alpha^{2} k^{2} \sigma_{0} \frac{\sin 2 k(b+d)}{\cosh ^{2} k d} \\
& \quad-\alpha \sigma_{1 b} \frac{\cosh k(b+d)}{\cosh k d} \cos (k a-\sigma t)-\alpha \sigma_{0} \sigma_{1 b} \frac{\cosh _{k}(b+d)}{\cosh k d} \sin (k a-\sigma t) \cdot t,
\end{aligned}
$$

For gravity waves of permanent form, the terms $t \cos (k a-\sigma t)$ and $t \sin (k a-\sigma t)$ that increase linearly with time have to be zero to avoid resonance. Noting that $\sigma_{1 b}=0$ or $\sigma_{1}=w_{1}=$ constant, then the general solution which satisfies the bottom boundary condition can be written as

$$
\begin{aligned}
f_{2}= & -\beta_{2} \frac{\cosh 2 k(b+d)}{\cosh ^{2} k d} \sin 2(k a-\sigma t)+\frac{1}{4} \alpha^{2} k \frac{\sin 2(k a-\sigma t)}{\cosh ^{2} k d} \\
& -\lambda_{2} \frac{\cosh k(b+d)}{\cosh k d} \sin (k a-\sigma t), \\
f_{2}^{\prime}= & \frac{1}{2} \alpha^{2} k \frac{\cosh 2 k(b+d)}{\cosh ^{2} k d} \sigma_{0} t, \\
g_{2}= & \beta_{2} \frac{\sinh 2 k(b+d)}{\cosh ^{2} k d} \cos 2(k a-\sigma t)+\lambda_{2} \frac{\sinh k(b+d)}{\cosh k d} \cos (k a-\sigma t), \\
g_{2}^{\prime}= & \frac{1}{4} \alpha^{2} k \frac{\sinh ^{2} k(b+d)}{\cosh ^{2} k d},
\end{aligned}
$$

Substituting (3.22) into the irrotational equation (2.4) in $\epsilon^{2}$ order, we obtain

$$
\begin{aligned}
\phi_{2 a}= & \sigma_{0}\left[2 \beta_{2} \frac{\cosh 2 k(b+d)}{\cosh ^{2} k d} \cos 2(k a-\sigma t)-\alpha^{2} k \frac{\cos 2(k a-\sigma t)}{\cosh ^{2} k d}\right] \\
& +\left(\alpha w_{1}+\sigma_{0} \lambda_{2}\right) \frac{\cosh k(b+d)}{\cosh k d} \cos (k a-\sigma t)-\phi_{2 a}^{\prime}, \\
\phi_{2 b}= & 2 \sigma_{0} \beta_{2} \frac{\sinh 2 k(b+d)}{\cosh ^{2} k d} \sin 2(k a-\sigma t) \\
& +\left(\alpha w_{1}+\sigma_{0} \lambda_{2}\right) \frac{\sinh k(b+d)}{\cosh k d} \sin (k a-\sigma t)-\phi_{2 b}^{\prime},
\end{aligned}
$$

Note that the secular terms in Eq. (3.23) have to be eliminated, which yields $\alpha w_{1}+\sigma_{0} \lambda_{2}=0$. The second-order Lagrangian velocity potential is obtained by integrating over the Lagrangian variables $a$ or $b$ as

$$
\begin{aligned}
& \phi_{2}=\frac{\sigma_{0}}{k} \beta_{2} \frac{\cosh 2 k(b+d)}{\cosh ^{2} k d} \sin 2(k a-\sigma t)-\frac{1}{2} \alpha^{2} \sigma_{0} \frac{\sin 2(k a-\sigma t)}{\cosh ^{2} k d}, \\
& \phi_{2}^{\prime}=D_{2}^{\prime}\left(\sigma_{0} t\right),
\end{aligned}
$$


Substituting the solutions up to the second order into the energy equation (3.18) in $\epsilon^{2}$ order, we can get

$$
\begin{aligned}
\frac{p_{2}}{\rho}= & \left\{\beta_{2}\left[2 \frac{\sigma_{0}^{2}}{k} \frac{\cosh 2 k(b+d)}{\cosh ^{2} k d}-g \frac{\sinh 2 k(b+d)}{\cosh ^{2} k d}\right]-\frac{3}{4} \alpha^{2} \sigma_{0}^{2} \frac{1}{\cosh ^{2} k d}\right\} \cos 2(k a-\sigma t) \\
& +\left[\alpha \frac{\sigma_{0}^{2}}{k} w_{1} \frac{\cosh k(b+d)}{\cosh k d}-g \lambda_{2} \frac{\sinh k(b+d)}{\cosh k d}\right] \cos (k a-\sigma t) \\
& +\left[\frac{1}{4} \alpha^{2} \sigma_{0}^{2} \frac{\cosh 2 k(b+d)}{\cosh ^{2} k d}-\frac{1}{4} g \alpha^{2} k \frac{\sinh 2 k(b+d)}{\cosh ^{2} k d}\right]-\sigma_{0} D^{\prime}{ }_{2 \sigma_{0} t} \\
& -\frac{T}{\rho}\left\{\left(4 k^{2} \beta_{2}+\frac{3}{4} \alpha^{2} k^{3}\right) \frac{\sinh 2 k(b+d)}{\cosh ^{2} k d} \cos 2(k a-\sigma t)+\frac{1}{4} \alpha^{2} k^{3} \frac{\sinh 2 k(b+d)}{\cosh ^{2} k d}\right. \\
& \left.+k \lambda_{2} \frac{\sinh k(b+d)}{\cosh k d} \cos (k a-\sigma t)\right\},
\end{aligned}
$$

Applying the zero pressure condition at the free surface, the unknown coefficients in Eq. (3.25) are obtained as

$$
\begin{gathered}
w_{1}=\lambda_{2}=0, \quad \phi_{2}^{\prime}=D_{2}^{\prime}\left(\sigma_{0} t\right)=\frac{1}{4} \alpha^{2} \sigma_{0}^{2}\left(\tanh ^{2} k d-1\right) t \\
\beta_{2}=\frac{3}{4} \frac{\alpha^{2} k}{2 \sigma_{0}^{2}-g k\left(1+\frac{4 T k^{2}}{\rho g}\right) \tanh 2 k d}\left(\sigma_{0}^{2} \frac{1}{\cosh 2 k d}+\frac{T}{\rho} k^{3} \tanh 2 k d\right),
\end{gathered}
$$

The second-order Lagrangian solutions are assembled as

$$
\begin{gathered}
f_{2}=\left[-\beta_{2} \frac{\cosh 2 k(b+d)}{\cosh ^{2} k d}+\frac{1}{4} \alpha^{2} k\left(1-\tanh ^{2} k d\right)\right] \sin 2(k a-\sigma t), \\
f_{2}^{\prime}=\frac{1}{2} \alpha^{2} k\left(1+\tanh ^{2} k d\right) \frac{\cosh 2 k(b+d)}{\cosh 2 k d} \sigma_{0} t \\
g_{2}=\beta_{2} \frac{\sinh 2 k(b+d)}{\cosh ^{2} k d} \cos 2(k a-\sigma t)+\frac{1}{4} \alpha^{2} k\left(1+\tanh ^{2} k d\right) \frac{\sinh 2 k(b+d)}{\cos k 2 k d}, \\
g_{2}^{\prime}=\frac{1}{4} \alpha^{2} k \frac{\sinh 2 k(b+d)}{\cosh ^{2} k d}, \\
\phi_{2}=\frac{\sigma_{0}}{k} \beta_{2}\left(1+\tanh ^{2} k d\right) \frac{\cosh 2 k(b+d)}{\cosh 2 k d} \sin 2(k a-\sigma t) \\
-\frac{1}{2} \alpha^{2} \sigma_{0}\left(1-\tanh ^{2} k d\right) \sin ^{2}(k a-\sigma t) \\
\phi^{\prime}{ }_{2}=\frac{1}{4} \alpha^{2} \sigma_{0}^{2}\left(\tanh ^{2} k d-1\right) t
\end{gathered}
$$

The horizontal particle trajectory in the second-order approximation includes a periodic component $f_{2}$ and a non-periodic function $f_{2}^{\prime}$ that increases linearly with time and represents the mass transport. This implies that, on average, a fluid particle moves forward and does not form a closed orbit as occurring in the first-order approximation. The vertical trajectory $y$ in this order includes a second harmonic component $g_{2}$ and a term $g_{2}^{\prime}$ that is a function of $b$ and independent of time. This second-order vertical mean level $g_{2}^{\prime}$ for a particle decays with the water depth. Equation (3.30) also confirms that the Lagrangian mean level of gravity waves is higher than the Eulerian mean level [32]. Unlike Longuet-Higgins [32] who used the Euler-Lagrange transformation to derive the above result, the present theory is perfectly constructed in the Lagrangian framework. For the limiting case $T=0$, one can verify that the present theory reduces to pure progressive waves of constant depth, as was previously obtained by Chen et al. [2]. 


\subsection{Third-Order Approximation}

The third-order governing equations and boundary conditions can be obtained by collecting the terms of $O\left(\epsilon^{3}\right)$

$$
\begin{aligned}
& f_{3 a}+f_{3 a}^{\prime}+g_{3 b}+g_{3 b}^{\prime}+f_{1 a}\left(g_{2 b}+g_{2 b}^{\prime}\right)+f_{2 a} g_{1 b}-f_{1 b} g_{2 a} \\
& -\left(f_{2 b}+f_{2 b}^{\prime}\right) g_{1 a}+\left(\sigma_{2 a} f_{1 \sigma t}+\sigma_{2 b} g_{1 \sigma t}\right) t=0, \\
& \sigma_{0}\left(f_{3 a \sigma t}+f_{3 a \sigma_{0} t}^{\prime}+g_{3 b \sigma t}+g_{3 b \sigma_{0} t}^{\prime}\right)+\sigma_{2}\left(f_{1 a \sigma t}+g_{1 b \sigma t}\right)+\sigma_{2 a} f_{1 \sigma t} \\
& +\sigma_{2 b} g_{1 \sigma t}+\sigma_{0}\left[\sigma_{2 a} f_{1(\sigma t)^{2}}+\sigma_{2 b} g_{1(\sigma t)^{2}}\right] t+\sigma_{0}\left[f_{1 a \sigma t} g_{2 b}\right. \\
& +f_{1 a \sigma t} g_{2 b}^{\prime}+f_{1 a} g_{2 b \sigma t}+f_{2 a \sigma t} g_{1 b}+f_{2 a} g_{1 b \sigma t}-f_{1 b \sigma t} g_{2 a}-f_{1 b} g_{2 a \sigma t} \\
& -\left(f_{2 b \sigma t}+f_{2 b \sigma_{0} t}^{\prime}\right) g_{1 a}-\left(f_{2 b}+f_{2 b}^{\prime}\right) g_{1 a \sigma t}=0, \\
& \sigma_{0}\left(f_{3 b \sigma t}+f_{3 b \sigma_{0} t}^{\prime}-g_{3 a \sigma t}-g_{3 a \sigma_{0} t}^{\prime}\right)+\sigma_{2}\left(f_{1 b \sigma t}-g_{1 a \sigma t}\right)+\sigma_{2 b} f_{1 \sigma t} \\
& -\sigma_{2 a} g_{1 \sigma t}+\sigma_{0}\left[\sigma_{2 b} f_{1(\sigma t)^{2}}-\sigma_{2 a} g_{1(\sigma t)^{2}}\right] t+\sigma_{0}\left[f_{1 a}\left(f_{2 b \sigma t}+f_{2 b \sigma_{0} t}^{\prime}\right)\right. \\
& +f_{2 a} f_{1 b \sigma t}-f_{2 a \sigma t} f_{1 b}-f_{1 a \sigma t}\left(f_{2 b}+f_{2 b}^{\prime}\right)+g_{1 a} g_{2 b \sigma t} \\
& \left.+g_{2 a} g_{1 b \sigma t}-g_{1 b} g_{2 a \sigma t}-g_{2 b} g_{1 a \sigma t}\right]=0, \\
& \phi_{3 a}+\phi_{3 a}^{\prime}=\sigma_{0}\left(f_{3 \sigma t}+f_{3 \sigma_{0} t}^{\prime}\right)+\sigma_{2} f_{1 \sigma t}+\sigma_{0}\left[f_{1 \sigma t} f_{2 a}\right. \\
& \left.+\left(f_{2 \sigma t}+f_{2 \sigma_{0} t}^{\prime}\right) f_{1 a}+g_{2 a} g_{1 \sigma t}+g_{1 a} g_{2 \sigma t}\right]-\sigma_{2 a} \phi_{1 \sigma t} t, \\
& \phi_{3 b}+\phi_{3 b}^{\prime}=\sigma_{0}\left(g_{3 \sigma t}+g_{3 \sigma_{0} t}^{\prime}\right)+\sigma_{2} g_{1 \sigma t}+\sigma_{0}\left[f _ { 1 \sigma t } \left(f_{2 b}\right.\right. \\
& \left.\left.+f_{2 b}^{\prime}\right)+\left(f_{2 \sigma t}+f_{2 \sigma_{0} t}^{\prime}\right) f_{1 b}+g_{2 b} g_{1 \sigma t}+g_{1 b} g_{2 \sigma t}\right]-\sigma_{2 b} \phi_{1 \sigma t} t, \\
& \frac{p_{3}}{\rho}=-\left[\sigma_{0}\left(\phi_{3 \sigma t}+\phi_{3 \sigma_{0} t}^{\prime}\right)+g\left(g_{3}+g_{3}^{\prime}\right)\right]-\sigma_{2} \phi_{1 \sigma t}+\sigma_{0}^{2}\left[f _ { 1 \sigma t } \left(f_{2 \sigma t}\right.\right. \\
& \left.\left.\left.+f_{2 \sigma_{0} t}^{\prime}\right)+g_{1 \sigma t} g_{2 \sigma t}\right)\right]+\frac{T}{\rho}\left\{\left(g_{3 a a}+g_{3 a a}^{\prime}\right)+\left(\sigma_{2 a} t g_{1 \sigma t}\right)_{a}-2 f_{1 a} g_{2 a a}\right. \\
& \left.-2 f_{2 a} g_{1 a a}-f_{2 a a} g_{1 a}-f_{1 a a} g_{2 a}+3 f_{1 a}^{2} g_{1 a a}+3 f_{1 a a} f_{1 a} g_{1 a}-\frac{3}{2} g_{1 a a} g_{1 a}^{2}\right\},
\end{aligned}
$$

On substituting the first- and second-order approximations into the governing equations (3.34)-(3.39), the third-order continuity, irrotational and energy equations become

$$
\begin{aligned}
f_{3 a}+ & f_{3 a}^{\prime}+g_{3 b}+g_{3 b}^{\prime} \\
= & -f_{1 a} g_{2 b}-f_{2 a} g_{1 b}+f_{1 b} g_{2 a}+\left(f_{2 b}+f_{2 b}^{\prime}\right) g_{1 a}-\sigma_{2 b} g_{1 \sigma t} t \\
= & \alpha k^{2}\left(2 \beta_{2}+\frac{1}{4} \alpha^{2} k\right) \frac{\cosh 3 k(b+d)}{\cosh ^{3} k d} \cos (k a-\sigma t) \\
& +\alpha k^{2}\left(2 \beta_{2}-\frac{1}{4} \alpha^{2} k\right) \frac{\cosh k(b+d)}{\cosh ^{3} k d} \cos 3(k a-\sigma t) \\
& -\alpha\left[\alpha^{2} k^{3} \sigma_{0} \frac{\sinh 2 k(b+d)}{\cosh ^{2} k d}+\sigma_{2 b}\right] \frac{\sinh k(b+d)}{\cosh k d} \sin (k a-\sigma t) \cdot t,
\end{aligned}
$$




$$
\begin{aligned}
& \sigma_{0}\left(f_{3 a \sigma t}+f_{3 a \sigma_{0} t}^{\prime}+g_{3 b \sigma t}+g_{3 b \sigma_{0} t}^{\prime}\right) \\
& =\alpha k^{2} \sigma_{0}\left[\left(2 \beta_{2}+\frac{1}{4} \alpha^{2} k\right) \frac{\cosh 3 k(b+d)}{\cosh ^{3} k d} \sin (k a-\sigma t)\right. \\
& \left.+\left(6 \beta_{2}-\frac{3}{4} \alpha^{2} k\right) \frac{\cosh k(b+d)}{\cosh ^{3} k d} \sin 3(k a-\sigma t)\right]-\alpha\left[\alpha^{2} k^{3} \sigma_{0} \frac{\sinh 2 k(b+d)}{\cosh ^{2} k d}+\sigma_{2 b}\right] \\
& \times \frac{\sinh k(b+d)}{\cosh k d} \sin (k a-\sigma t)+\alpha \sigma_{0}\left[\alpha^{2} k^{3} \sigma_{0} \frac{\sinh 2 k(b+d)}{\cosh ^{2} k d}+\sigma_{2 b}\right] \frac{\sinh k(b+d)}{\cosh k d} \cos (k a-\sigma t) \cdot t, \\
& \sigma_{0}\left(f_{3 b \sigma t}+f^{\prime}{ }_{3 b \sigma_{0} t}-g_{3 a \sigma t}-g^{\prime}{ }_{3 a \sigma_{0} t}\right) \\
& =\alpha k^{2} \sigma_{0}\left[\left(6 \beta_{2}+\frac{3}{4} \alpha^{2} k\right) \frac{\sinh 3 k(b+d)}{\cosh ^{3} k d} \cos (k a-\sigma t)\right. \\
& \left.+\left(2 \beta_{2}+\frac{1}{4} \alpha^{2} k\right) \frac{\sinh k(b+d)}{\cosh ^{3} k d} \cos 3(k a-\sigma t)\right]-\alpha\left[\frac{1}{2} \alpha^{2} k^{3} \sigma_{0} \frac{\sinh k(b+d)}{\cosh ^{3} k d}\right. \\
& \left.+\sigma_{2 b} \frac{\cosh k(b+d)}{\cosh k d}\right] \cos (k a-\sigma t)-\alpha \sigma_{0}\left[\alpha^{2} k^{3} \sigma_{0} \frac{\sinh 2 k(b+d)}{\cosh ^{2} k d}+\sigma_{2 b}\right] \frac{\cosh k(b+d)}{\cosh k d} \sin (k a-\sigma t) \cdot t,
\end{aligned}
$$

From Eqs. (3.40)-(3.42), the secular terms that grow with time have to be zero. We can obtain

$$
\sigma_{2 a}=0, \quad \sigma_{2 b}=-\alpha^{2} k^{3} \sigma_{o} \frac{\sinh 2 k(b+d)}{\cosh ^{2} k d} .
$$

Integrating Eq. (3.43) with $b, \sigma_{2}$ is given by

$$
\sigma_{2}=-\frac{1}{2} \alpha^{2} k^{3} \frac{\cosh 2 k(b+d)}{\cosh ^{2} k d} \sigma_{o}+w_{2},
$$

where omega is a constant which needs to be solved.

Using Eq. (3.43), Eqs. (3.40)-(3.42) can be reduced to

$$
\begin{aligned}
& f_{3 a}+f_{3 a}^{\prime}+g_{3 b}+g_{3 b}^{\prime}=\alpha k^{2}\left(2 \beta_{2}+\frac{1}{4} \alpha^{2} k\right) \frac{\cosh 3 k(b+d)}{\cosh ^{3} k d} \cos (k a-\sigma t) \\
& +\alpha k^{2}\left(2 \beta_{2}-\frac{1}{4} \alpha^{2} k\right) \frac{\cosh k(b+d)}{\cosh ^{3} k d} \cos 3(k a-\sigma t), \\
& \sigma_{o}\left(f_{3 a \sigma t}+f_{3 a \sigma_{0} t}^{\prime}+g_{3 b \sigma t}+g_{3 b \sigma_{0} t}^{\prime}\right) \\
& =\alpha k^{2} \sigma_{o}\left[\left(2 \beta_{2}+\frac{1}{4} \alpha^{2} k\right) \frac{\cosh 3 k(b+d)}{\cosh ^{3} k d} \sin (k a-\sigma t)\right. \\
& \left.\quad+\left(6 \beta_{2}-\frac{3}{4} \alpha^{2} k\right) \frac{\cosh k(b+d)}{\cosh ^{3} k d} \sin 3(k a-\sigma t)\right] \\
& \sigma_{o}\left(f_{3 b \sigma t}+f_{3 b \sigma_{0} t}^{\prime}-g_{3 a \sigma t}-g_{3 a \sigma_{0} t}^{\prime}\right) \\
& =\alpha k^{2} \sigma_{o}\left[\left(6 \beta_{2}+\frac{5}{4} \alpha^{2} k\right) \frac{\sinh ^{2} k(b+d)}{\cosh ^{3} k d} \cos (k a-\sigma t)\right. \\
& \left.\quad+\left(2 \beta_{2}+\frac{1}{4} \alpha^{2} k\right) \frac{\sinh ^{2}(b+d)}{\cosh ^{3} k d} \cos 3(k a-\sigma t)\right]
\end{aligned}
$$


From Eqs. (3.45)-(3.47), the solutions of $f_{3}, f_{3}^{\prime}, g_{3}$ and $g_{3}^{\prime}$ can be assumed as

$$
\begin{aligned}
f_{3}= & {\left[-\beta_{3} \frac{\cosh 3 k(b+d)}{\cosh ^{3} k d}+\frac{1}{6} \alpha k\left(5 \beta_{2}-\frac{1}{2} \alpha^{2} k\right) \frac{\cosh k(b+d)}{\cosh ^{3} k d}\right] \sin 3(k a-\sigma t) } \\
& -\left[\frac{1}{2} \alpha k\left(5 \beta_{2}+\alpha^{2} k\right) \frac{\cosh 3 k(b+d)}{\cosh ^{3} k d}+\lambda_{3} \frac{\cosh k(b+d)}{\cosh ^{3} k d}\right] \sin (k a-\sigma t), \\
f_{3}^{\prime}= & 0, \\
g_{3}= & {\left[\beta_{3} \frac{\sinh 3 k(b+d)}{\cosh ^{3} k d}-\frac{1}{2} \alpha k \beta_{2} \frac{\sinh k(b+d)}{\cosh ^{3} k d}\right] \sin 3(k a-\sigma t) } \\
& +\left[\frac{1}{2} \alpha k\left(3 \beta_{2}+\frac{1}{2} \alpha^{2} k\right) \frac{\sinh 3 k(b+d)}{\cosh ^{3} k d}+\lambda_{3} \frac{\sinh k(b+d)}{\cosh ^{3} k d}\right] \cos (k a-\sigma t), \\
g_{3}^{\prime}= & 0 .
\end{aligned}
$$

where $\beta_{3}$ and $\lambda_{3}$ are undetermined coefficients which can be found by using the dynamic free surface boundary condition. Substituting these terms, the first- and the second-order solutions into Eqs. (3.37) and (3.38), we can get

$$
\begin{aligned}
& \phi_{3 a}+\phi_{3 a}^{\prime} \\
&=3 \sigma_{0}\left[\beta_{3} \frac{\cosh 3 k(b+d)}{\cosh ^{3} k d}-\frac{1}{2} \alpha k\left(3 \beta_{2}-\frac{1}{2} \alpha^{2} k\right) \frac{\cosh k(b+d)}{\cosh ^{3} k d}\right] \cos 3(k a-\sigma t) \\
&+\sigma_{0}\left[\frac{1}{2} \alpha k \beta_{2} \frac{\cosh 3 k(b+d)}{\cosh ^{3} k d}+\left(\alpha \frac{w_{2}}{\sigma_{0}}+\lambda_{3} \operatorname{sech}^{2} k d\right) \frac{\cosh k(b+d)}{\cosh k d}\right] \cos (k a-\sigma t), \\
& \phi_{3 b}+\phi_{3 b}^{\prime}=3 \sigma_{o}\left[\beta_{3} \sin 3(k a-\sigma t)+\frac{1}{2} \alpha k \beta_{2} \sin (k a-\sigma t)\right] \frac{\sinh 3 k(b+d)}{\cosh ^{3} k d} \\
&+\sigma_{0}\left[-\frac{1}{2} \alpha k\left(3 \beta_{2}-\frac{1}{2} \alpha^{2} k\right) \sin 3(k a-\sigma t)+\left(\alpha \frac{w_{2}}{\sigma_{0}} \cosh ^{2} k d+\lambda_{3}\right)\right. \\
&\quad \times \sin (k a-\sigma t)] \frac{\sinh 3 k(b+d)}{\cosh ^{3} k d},
\end{aligned}
$$

From Eqs. (3.52) and (3.53), we get

$$
\begin{aligned}
\phi_{3}= & \frac{\sigma_{0}}{k} \beta_{3} \frac{\cosh 3 k(b+d)}{\cosh ^{3} k d} \sin 3(k a-\sigma t)+\frac{1}{2} \alpha \beta_{2} \sigma_{0} \frac{\cosh 3 k(b+d)}{\cosh ^{3} k d} \\
& \times \sin (k a-\sigma t)-\frac{1}{2} \alpha\left(3 \beta_{2}-\frac{1}{2} \alpha^{2} k\right) \sigma_{0} \frac{\cosh k(b+d)}{\cosh ^{3} k d} \sin 3(k a-\sigma t)+D_{3},
\end{aligned}
$$

and

$$
\phi_{3}^{\prime}=D_{3}^{\prime}\left(\sigma_{0} t\right), \alpha w_{2}+\sigma_{0} \lambda_{3} \operatorname{sech}^{2} k d=0
$$


The wave pressure can thus be given by

$$
\begin{aligned}
\frac{p_{3}}{\rho}= & \left\{\beta_{3}\left[3 \frac{\sigma_{0}^{2}}{k} \frac{\cosh 3 k(b+d)}{\cosh ^{3} k d}-g \frac{\sinh 3 k(b+d)}{\cosh ^{3} k d}\right]-\frac{1}{2} \alpha\left(7 \beta_{2}-\alpha^{2} k\right) \sigma_{0}^{2} \frac{\cosh k(b+d)}{\cosh ^{3} k d}\right. \\
& \left.+\frac{1}{2} \alpha k g \beta_{2} \frac{\sinh k(b+d)}{\cosh ^{3} k d}\right\} \cos 3(k a-\sigma t)-\sigma_{0} D_{3 \sigma_{0} t}^{\prime} \\
& +\left\{\frac{3}{2} \alpha \beta_{2} \sigma_{0}^{2} \frac{\cosh 3 k(b+d)}{\cosh ^{3} k d}-\frac{1}{2} \alpha k g\left(3 \beta_{2}+\frac{1}{2} \alpha^{2} k\right) \frac{\sinh 3 k(b+d)}{\cosh ^{3} k d}\right. \\
& \left.+\left(\sigma_{0} \frac{\alpha}{k} w_{2} \cosh ^{2} k d-\frac{1}{4} \alpha^{3} k \sigma_{0}^{2}\right) \frac{\cosh k(b+d)}{\cosh ^{3} k d}-g \lambda_{3} \frac{\sinh k(b+d)}{\cosh ^{3} k d}\right\} \cos (k a-\sigma t) \\
& +\frac{T}{\rho}\left(-9 k^{3} \beta_{3}-\frac{9}{2} k^{3} \alpha \beta_{2}-\frac{15}{32} k^{4} \alpha^{3}\right) \frac{\sinh ^{2}(b(b+d)}{\cosh { }^{3} k d} \cos 3(k a-\sigma t) \\
& -\left(3 k^{3} \alpha \beta_{2}+\frac{17}{32} k^{4} \alpha^{3}\right) \frac{\sinh ^{3} k(b+d)}{\cosh ^{3} k d} \cos (k a-\sigma t) \\
& +\left(4 k^{3} \alpha \beta_{2}+\frac{29}{32} k^{4} \alpha^{3}\right) \frac{\sinh ^{2}(b+d)}{\cosh ^{3} k d} \cos 3(k a-\sigma t) \\
& +\left(-k^{2} \lambda_{3}-\frac{3}{2} k^{3} \alpha \beta_{2}-\frac{21}{32} k^{4} \alpha^{3}\right) \frac{\sinh k(b+d)}{\cosh { }^{3} k d} \cos (k a-\sigma t)
\end{aligned}
$$

The procedure to obtain the solutions at this order is similar to that of $O\left(\epsilon^{2}\right)$. The secular terms that grow with time have to be zero. Using zero pressure condition at the free surface $\left(p_{3}=0\right.$ at $\left.b=0\right)$, we obtain

$$
\begin{aligned}
\beta_{3}[ & \left.3 \frac{\sigma_{0}^{2}}{k} \frac{\cosh 3 k d}{\cosh ^{3} k d}-g \frac{\sinh 3 k d}{\cosh ^{3} k d}\right] \\
& -\frac{1}{2} \alpha\left(7 \beta_{2}-\alpha^{2} k\right) \sigma_{0}^{2} \frac{\cosh k d}{\cosh ^{3} k d}-\frac{T}{\rho}\left(\frac{9}{2} k^{3} \alpha \beta_{2}+\frac{15}{32} k^{4} \alpha^{3}+9 k^{2} \beta_{3}\right) \frac{\sinh 3 k d}{\cosh ^{3} k d} \\
& +\left[\frac{1}{2} \alpha k g \beta_{2}+\frac{T}{\rho}\left(4 k^{3} \alpha \beta_{2}+\frac{29}{32} k^{4} \alpha^{3}\right)\right] \frac{\sinh k d}{\cosh ^{3} k d}=0,
\end{aligned}
$$

From Eq. (3.57), we get the solution of $\beta_{3}$

$$
\begin{aligned}
\beta_{3}= & \frac{k \alpha}{\left(48 \rho \sigma^{2} \cosh 3 k d-16 \rho g k\left(1+\frac{9 T k^{3}}{\rho g}\right) \sinh 3 k d\right)}\left[-8\left(k \alpha^{2}-7 \beta_{2}\right) \rho \sigma_{0}^{2} \cosh k d\right. \\
& \left.+k\left(-7 T k^{3} \alpha^{2}-8 \rho g \beta_{2}\left(1-\frac{T k^{2}}{\rho g}\right)+3 k^{2} T\left(5 k \alpha^{2}+48 \beta_{2}\right) \cosh 2 k d\right) \sinh k d\right]
\end{aligned}
$$

and

$$
\begin{aligned}
& \frac{3}{2} \alpha \beta_{2} \sigma_{0}^{2} \frac{\cosh 3 k d}{\cosh ^{3} k d}-\frac{1}{2} \alpha k g\left(3 \beta_{2}+\frac{1}{2} \alpha^{2} k\right) \frac{\sinh 3 k d}{\cosh ^{3} k d}+\left(\sigma_{0} \frac{\alpha}{k} w_{2} \cosh ^{2} k d\right. \\
& \left.-\frac{1}{4} \alpha^{3} k \sigma_{0}^{2}\right) \frac{\cosh k d}{\cosh ^{3} k d}-g \lambda_{3} \frac{\sinh k d}{\cosh ^{3} k d}+\frac{T}{\rho}\left[\left(-3 k^{3} \alpha \beta_{2}-\frac{17}{32} k^{4} \alpha^{3}\right) \frac{\sinh 3 k d}{\cosh ^{3} k d}\right. \\
& \left.\quad-\left(k^{2} \lambda_{3}+\frac{3}{2} k^{3} \alpha \beta_{2}+\frac{21}{32} k^{4} \alpha^{3}\right) \frac{\sinh k d}{\cosh ^{3} k d}\right]=0
\end{aligned}
$$


From Eq. (3.59), we can obtain the solution of $\lambda_{3}$.

$$
\begin{aligned}
\lambda_{3}= & \frac{k^{2} \alpha^{3}}{\left.64\left(k^{2} T+\rho g\right)\left(4 k^{2} T+\rho g+\left(2 k^{2} T-g \rho\right) \cosh 2 k d\right)\right)}\left[106 k^{4} T^{2}+179 g \rho k^{2} T\right. \\
& \left.+64 g^{2} \rho^{2}+60 k^{2} T\left(k^{2} T+\rho g\right) \cosh 2 k d+\left(2 k^{4} T^{2}+\rho g k^{2} T+8 \rho^{2} g^{2}\right) \cosh 4 k d\right] .
\end{aligned}
$$

From Eq. (3.55), the solution of $w_{2}$ is

$$
w_{2}=-\frac{\sigma_{0}}{\alpha} \lambda_{3} \operatorname{sech}^{2} k d
$$

Finally, the physical parameters to the third-order solutions in Lagrangian form are given. Equation (3.60) is the second-order angular frequency correction for a particle, in which the first term is the second-order Stokes wave frequency and the second term varies monotonically with the vertical label $b$ or the wavelength-averaged level of the particles. The third-order solutions of Eqs. (3.48)-(3.51) are periodic functions and have a combination of both first and third harmonic components. Thus, the solution of system has the following expressions:

$$
\begin{aligned}
& x=x(a, b, t)=a+f_{1}+f_{2}+f_{2}^{\prime}+f_{3}, \\
& y=y(a, b, t)=b+g_{1}+g_{2}+g_{2}^{\prime}+g_{3}, \\
& \phi=\phi(a, b, t)=\phi_{1}+\phi_{2}+\phi_{3}, \\
& p=p(a, b, t)=-\rho g b+p_{1}+p_{2}+p_{3}, \\
& \sigma=\sigma(b)=\sigma_{0}+\sigma_{2}=2 \pi / T_{L}(b),
\end{aligned}
$$

The set of Eqs. (3.61)-(3.65) ensures that Bernoulli's condition of constant pressure is satisfied on the free surface.

\section{Further Results on the Fluid Motion}

\subsection{Mass Transport Velocity}

Taking time-average over one Lagrangian wave period in terms of the horizontal particle displacement, the so-called drift velocity over the whole range of depths can be obtained as follows:

$$
\begin{aligned}
\frac{\bar{x}_{t}}{c_{0}} & =\frac{\overline{\sum_{n=1}^{3} \epsilon^{n}\left[f_{n t}\left(a, b, \sigma_{L} t\right)+f_{n t}^{\prime}\left(a, b, \sigma_{L 0} t\right)\right]}}{c_{0}}=\frac{1}{2} \alpha^{2} k^{2}\left(1+\tanh ^{2} k d\right) \frac{\cosh 2 k(b+d)}{\cosh 2 k d}, \\
c_{0} & =\frac{\sigma_{0}}{k},
\end{aligned}
$$

where the overbar denotes time-average over a Lagrangian wave period, i.e. the period of particle motion, where $c_{0}$ is the linear phase speed. The first term of Eq. (4.1) on the right-hand side, a second-order correction quantity, is the same as that obtained by Longuet-Higgins [30] as $T=0$. From (4.1), the mass transport velocity is a function of the wave steepness, the water depth, the surface tension and the vertical Lagrangian label. Differentiating (4.1) with respect to the vertical Lagrangian label $b$ shows that the second-order drift velocity is always positive but monotonically decays with depth from the surface to the bottom. In Fig. 1 the mass transport velocity is plotted against the relative water depth $d / L=0.5$ and the relative wave height $k a=0.03 \pi$ for various Bond numbers $\left(\kappa=\frac{T k^{2}}{\rho g}=0,0.4,0.45\right)$ and vertical label $b$. It can be seen that the effect of increasing surface tension is generally to augment the magnitude and extent of the time-averaged drift velocity, thus resulting in large horizontal distance traveled by a particle compared with the case without surface tension. It is also remarkable that under the same wave subsurface particles travel slower and diminishes rapidly with the vertical position below the free surface. 


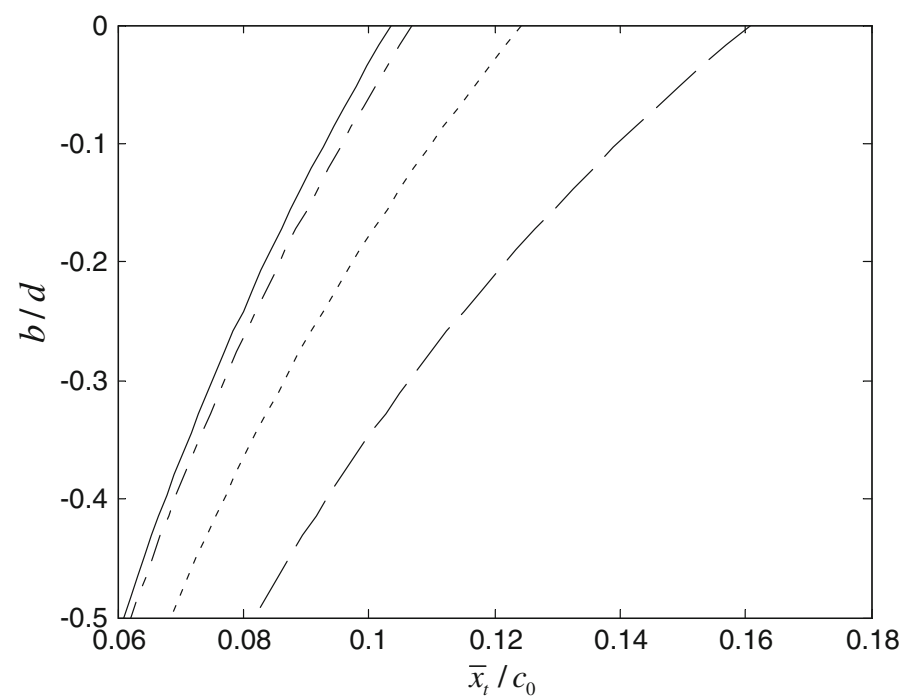

FIG. 1. Drift-velocity ratio as a function of the vertical label of fluid particles for gravity-capillary waves with various surface tension conditions $\kappa=\frac{T k^{2}}{\rho g}=0$ (solid line), 0.1 (dash-dotted line), 0.3 (dotted line), $0.4($ dash line), d/L=0.5,

$$
k a=0.03
$$

\subsection{Lagrangian Wave Frequency}

The Lagrangian angular frequency $\sigma$ up to third order can also be obtained as

$$
\begin{aligned}
\sigma & =\sigma_{0}+\sigma_{2}(b) \\
& =\sigma_{0}-\frac{1}{2} \alpha^{2} k^{2}\left(1+\tanh ^{2} k d\right) \frac{\cosh 2 k(b+d)}{\cosh 2 k d} \sigma_{o}-\sigma_{0} \frac{\lambda_{3}}{\alpha} \operatorname{sech}^{2} k d,
\end{aligned}
$$

Hence, a general Lagrangian wave period $\sigma$ differing from the Eulerian wave period for all particles at different vertical level $b$ can be obtained directly in the odd-order Lagrangian solutions. The difference between the Lagrangian frequency $\sigma$ and the Eulerian wave frequency $\sigma_{w}$, as well as the Eulerian wave frequency itself, are

$$
\begin{aligned}
\sigma-\sigma_{w} & =-\frac{1}{2} \alpha^{2} k^{2}\left(1+\tanh ^{2} k d\right) \frac{\cosh 2 k(b+d)}{\cosh 2 k d} \sigma_{0}, \\
\sigma_{w}=w_{2} & =\sigma_{0}-\sigma_{0} \frac{\lambda_{3}}{\alpha} \operatorname{sech}^{2} k d .
\end{aligned}
$$

where $\sigma_{w}$ is the angular frequency computed by Stokes expansion in the Eulerian system [35].

\subsection{Lagrangian Mean Level}

Averaging the particle elevation up to the third order over a given period of particle motion, the present theory gives the Lagrangian mean level $\bar{\eta}_{L}(b)$ which is higher than the Eulerian mean level $\bar{\eta}_{w}=0$ as

$$
\bar{\eta}_{L}-\bar{\eta}_{w}=\frac{1}{T_{L}} \int_{0}^{T_{L}} y \mathrm{~d} t=g_{2}^{\prime}=\frac{1}{4} \alpha^{2} k \frac{\sinh 2 k(b+d)}{\cosh ^{2} k d},
$$

Longuet-Higgins $[31,32]$ also showed that the Lagrangian mean level is higher than the Eulerian mean level for progressive water waves. However, his expression is applicable only to particles at the free surface and is the same as that given by the first term of Eq. (4.4) at $b=0$. 

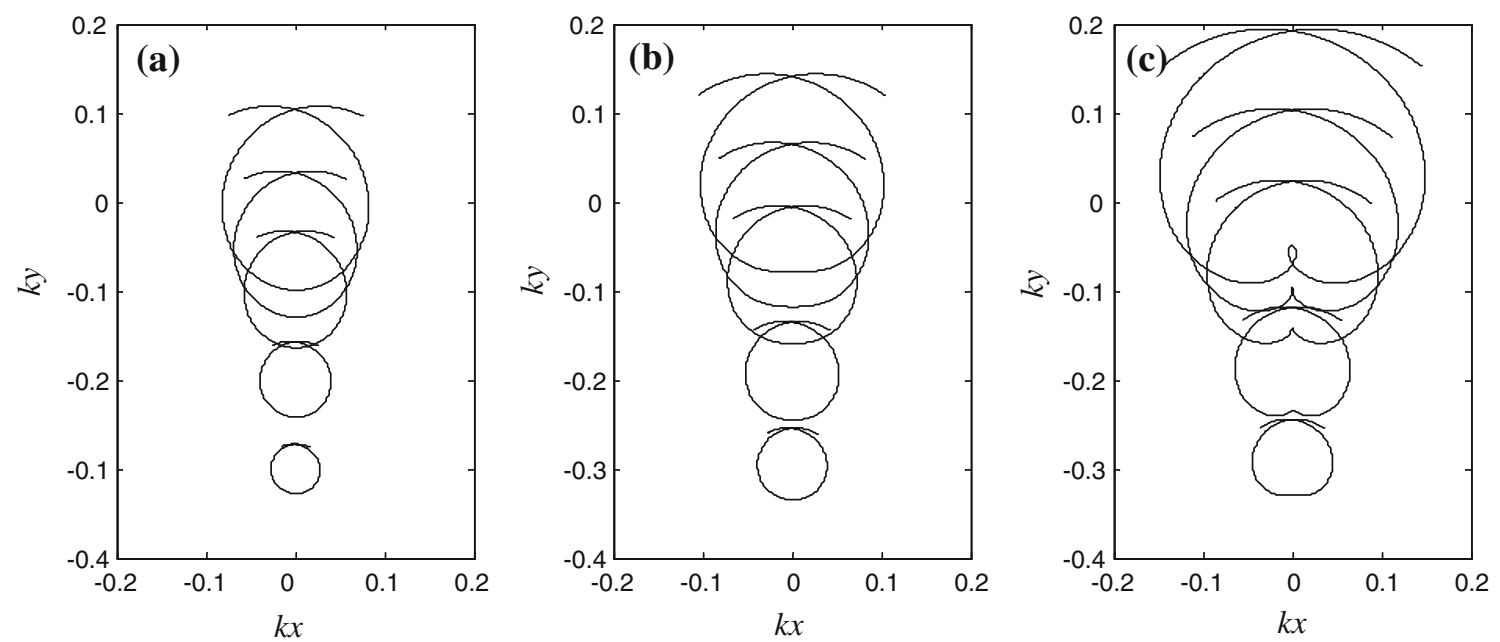

FIG. 2. Water particle orbits calculated from analytical solutions to a third-order approximation in Lagrangian form at different elevations $b$ for water depth $d / L=0.5$ and wave steepness $k a=0.03 \pi$ under various surface tension conditions.

$$
\mathbf{a} \kappa=\frac{T k^{2}}{\rho g}=0, \mathbf{b} \kappa=\frac{T k^{2}}{\rho g}=0.4, \mathbf{c} \kappa=\frac{T k^{2}}{\rho g}=0.45
$$

\subsection{The Particle Orbits}

The most important characteristic of fluid motion described by the Lagrangian solution is the trajectories of particles which are represented by Eqs. (3.61) and (3.62). The parameter $\alpha$ can be determined by the wave height $H$ defined as half the vertical distance between the wave crest and wave trough, wave number $k$ and the water depth $d$ given. Hence, we have

$$
\frac{H}{2}=\left[g_{1}+g_{3}\right]_{b=0, k a-\sigma t=2 n \pi}, \quad n \in I
$$

the horizontal and vertical particle trajectories are

$$
x=a+\sum_{n=1}^{3}\left(f_{n}+f_{n}^{\prime}\right), \quad y=b+\sum_{n=1}^{3}\left(g_{n}+g_{n}^{\prime}\right) .
$$

It is immediately obvious that the orbit of the particles is not a closed curve but a spiraling-progressive curve owing to the existence of a drift displacement persisting with it along the wave direction. In Figs. 2 and 3, the particle trajectories for gravity-capillary waves with different values of wave steepness $(k a=0.03 \pi, 0.05 \pi)$ in finite water depth have been plotted for several values of surface tension $\left(\kappa=\frac{T k}{\rho g}=0,0.4,0.45\right)$ and level $b$, including $b=0$ for particles at the free surface. While the absence of closed particle paths has been shown previously [18,19], the present approach extends the theory to nonlinear water waves and provides approximations that enable quantitative estimates. It can be seen in each of the plotted orbits that a water particle advances a distance forward which is commonly referred to as the mean horizontal drift or mass transport in the direction of wave propagation. The water particle at the free surface $(b=0)$ travels furthest, whilst that in the interior of the fluid propagates less and slower. To the third-order approximation, the particle trajectory has an open orbit, irrespective of their initial mean locations. The orbital shapes near the bottom are more elliptical since the vertical excursion of the particle is less than its horizontal counterpart. However, for the orbits near or at the still water level, the vertical excursion is greater. 

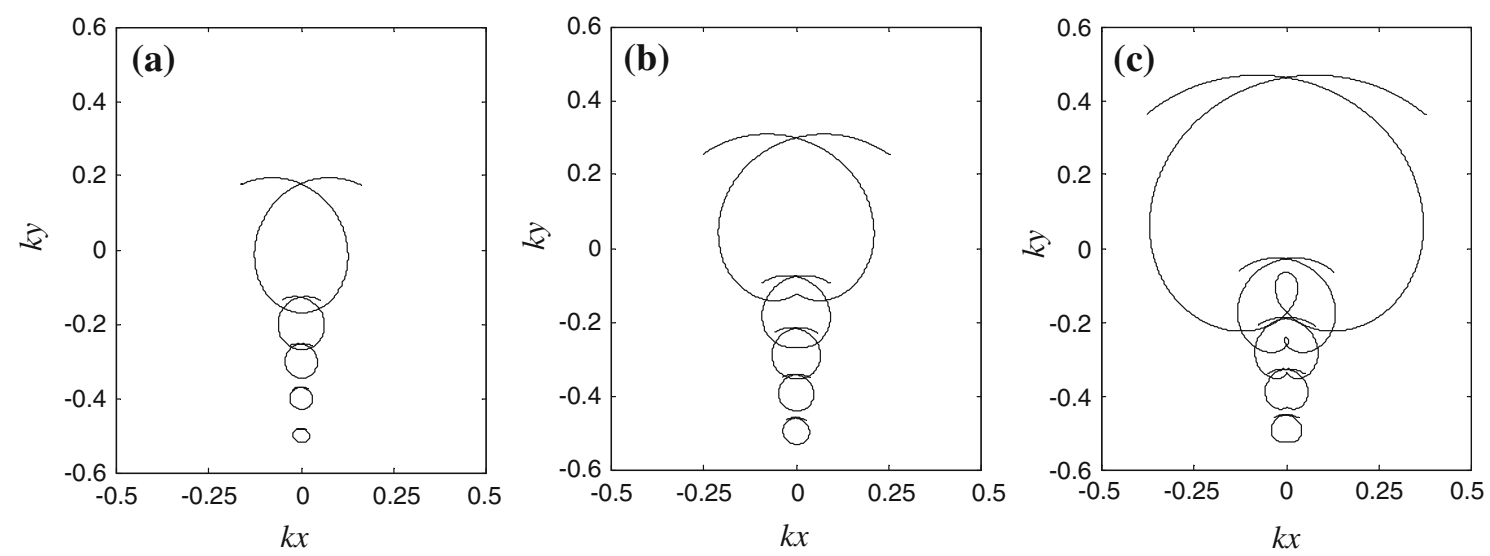

FIG. 3. Water particle orbits calculated from analytical solutions to a third-order approximation in Lagrangian form at different elevations $b$ for water depth $d / L=0.5$ and wave steepness $k a=0.05 \pi$ under various surface tension conditions.

$$
\mathbf{a} \kappa=\frac{T k^{2}}{\rho g}=0, \mathbf{b} \kappa=\frac{T k^{2}}{\rho g}=0.4, \mathbf{c} \kappa=\frac{T k^{2}}{\rho g}=0.45
$$

From Fig. 2, it can be seen that the surface particles of the wave most influenced by surface tension $(\kappa=0.45)$ travel further and faster than the wave with $\kappa=0$ and $\kappa=0.4$, where $\kappa=0$ corresponds to pure gravity water waves. In the case of gravity wave with surface tension, the effect of increasing surface tension is generally to augment the magnitude and extent of the time-averaged drift velocity, thus resulting in large horizontal distance traveled by a particle compared with the case without surface tension. Both the vertical and horizontal excursions of the water particles also increase with surface tension, where the horizontal excursion of the particle is actually less than the vertical excursion. Shown in Fig. $2 \mathrm{~b}$ is a trajectory cusped in the trough. In particular, the double-loop trajectory of the free-surface particles is remarkably shown in Fig. 2c, with the form persisting for some subsurface particles. Nevertheless, at greater depths it becomes circular. Overall, an increase in surface tension tends to increase the motions of water particles. For higher waves shown in Fig. 3, it could be expected that the cusps appeared in Fig. $2 \mathrm{~b}$ will become subloops and the double-loop become more noticeable as the wave steepness increases.

\section{Concluding Remarks}

A particle-specific description of irrotational finite-amplitude progressive gravity-capillary waves in water of uniform depth satisfying all the governing equations and the boundary conditions is presented. The new Lagrangian solution is obtained to the third order. It can be used not only to determine the wave properties available in the Eulerian solution, but also to get the trajectory, the period, the mass transport and the Lagrangian mean level of a water particle which are not available from the Eulerian solution. The results for the particle trajectories and mass transport velocity of nonlinear gravity waves influenced by the surface tension are presented. The effect of increasing surface tension is to produce an increase in the relative horizontal distance traveled by a surface particle in an orbit and generally to increase the magnitude and extent of the mass transport velocity. Under the conditions of large surface tension or higher waves, a free-surface particle may undergo an extraordinary trajectory with a subloop on a main loop, with the form persisting for some depth below the free surface. At greater depths, the trajectory becomes circular.

Acknowledgments. The authors would like to acknowledge the insightful critiquing of the two referees. 
Open Access. This article is distributed under the terms of the Creative Commons Attribution Noncommercial License which permits any noncommercial use, distribution, and reproduction in any medium, provided the original author(s) and source are credited.

\section{References}

[1] Chen, Y.Y., Hsu, H.C.: Third-order asymptotic solution of nonlinear standing water waves in Lagrangian coordinates. Chin. Phys. B. 18, 861 (2009)

[2] Chen, Y.Y., Hsu, H.C., Chen, G.Y.: Lagrangian experiment and solution for irrotational finite-amplitude progressive gravity waves at uniform depth. Fluid Dyn. Res. 42, 045511 (2010, in press)

[3] Constantin, A.: The trajectories of particles in Stokes waves. Invent. Math. 166, 523-535 (2006)

[4] Constantin, A., Ehrnström, M., Villari, G.: Particle trajectories in linear deep-water waves. Nonlinear Anal. 9, 1336$1344(2008)$

[5] Constantin, A., Escher, J.: Symmetry of steady deep-water waves with vorticity. Eur. J. Appl. Math. 15, 755-768 (2004)

[6] Constantin, A., Escher, J.: Symmetry of steady periodic surface water waves with vorticity. J. Fluid Mech. 498, 171-181 (2004)

[7] Constantin, A., Escher, J.: Particle trajectories in solitary water waves. Bull. Am. Math. Soc. 44, 423-431 (2007)

[8] Constantin, A., Escher, J.: Analyticity of periodic traveling free surface water waves with vorticity. Ann. Math. 172, $1-6(2010)$

[9] Constantin, A., Sattinger, D., Strauss, W.: Variational formulations for steady water waves with vorticity. J. Fluid Mech. 548, 151-163 (2006)

[10] Constantin, A., Strauss, W.: Exact periodic travelling water waves with vorticity. C.R. Math. Acad. Sci. Paris 335, 797-800 (2002)

[11] Constantin, A., Strauss, W.: Exact steady periodic water waves with vorticity. Commun. Pure Appl. Math. 57, 481-527 (2004)

[12] Constantin, A., Strauss, W.: Pressure beneath a Stokes wave. Commun. Pure Appl. Math. 63, 533-557 (2010)

[13] Constantin, A., Villari, G.: Particle trajectories in linear water waves. J. Math. Fluid Mech. 10, 1-18 (2008)

[14] Crapper, G.D.: An exact solution for progressive capillary waves of arbitrary amplitude. J. Fluid Mech. 2, 532$540(1957)$

[15] Ehrnström, M.: Uniqueness for steady periodic water waves with vorticity. Int. Math. Res. Not. 2005, 3721-3726 (2005)

[16] Ehrnström, M., Villari, G.: Linear water waves with vorticity: rotational features and particle paths. J. Differ. Equ. 244, 1888-1909 (2008)

[17] Henry, D.: The trajectories of particles in deep-water Stokes waves. Int. Math. Res. Not. 2006, 1-13 (2006)

[18] Henry, D.: Particle trajectories in linear periodic capillary and capillary-gravity deep-water waves. J. Nonlinear Math. Phys. 14, 1-7 (2007)

[19] Henry, D.: Particle trajectories in linear periodic capillary and capillary-gravity water waves. Phil. Trans. R. Soc. A 365, 2241-2251 (2007)

[20] Henry, D.: On the deep-water Stokes wave flow. Int. Math. Res. Not. 2008, 1-7 (2008)

[21] Henry, D.: Pressure in a deep-water Stokes wave. J. Math. Fluid Mech. doi:10.1007/s00021-009-0015-0

[22] Henry, D.: Analyticity of streamlines for periodic travelling free surface capillary-gravity water waves with vorticity. SIAM J. Math. Anal. (2010, to appear)

[23] Hogan, S.J.: Particle trajectories in nonlinear capillary waves. J. Fluid Mech. 143, 243-252 (1984)

[24] Hogan, S.J.: Particle trajectories in nonlinear gravity-capillary waves. J. Fluid Mech. 151, 105-119 (1985)

[25] Hsu, H.C., Chen, Y.Y., Wang, C.F.: Perturbation analysis of short-crested waves in Lagrangian coordinates. Nonlinear Anal. Real World Appl. 11(3), 1522-1536 (2010)

[26] Ionescu-Kruse, D.: Particle trajectories in linearized irrotational shallow water flows. J. Nonlinear Math. Phys. 15, 13-27 (2008)

[27] Ionescu-Kruse, D.: Particle trajectories beneath small amplitude shallow water waves in constant vorticity flows. Nonlinear Anal. 71, 3779-3793 (2009)

[28] Kinnersley, W.: Exact large amplitude capillary waves on sheets of fluids. J. Fluid Mech. 77, 229-241 (1976)

[29] Lamb, H.: Hydrodynamics, 6th edn. Cambridge University Press, Cambridge (1932)

[30] Longuet-Higgins, M.S.: Mass transport in water waves. Phil. Trans. R. Soc. A. 245, 533-581 (1953)

[31] Longuet-Higgins, M.S.: The trajectories of particles in steep, symmetric gravity waves. J. Fluid Mech. 94, 497-517 (1979)

[32] Longuet-Higgins, M.S.: Eulerian and Lagrangian aspects of surface waves. J. Fluid Mech. 173, 683-707 (1986)

[33] Mei, C.C.: The Applied Dynamics of Ocean Surface Waves. Wiley, New York (1983)

[34] Miche, A.: Mouvements ondulatoires de la mer en profondeur constante ou décroissante. Annales des ponts et chaussees. 25-78, 131-164, 270-292, 369-406 (1944)

[35] Nayfeh, A.H.: Finite amplitude surface waves in a liquid layer. J. Fluid Mech. 40, 671-684 (1970)

[36] Piedra-Cueva, I.: Drift velocity of spatially decaying waves in a two-layer viscous system. J. Fluid Mech. 229, 217-239 (1995)

[37] Pierson, W.J.: Perturbation analysis of the Navier-Stokes equations in Lagrangian form with selected linear solution. J. Geophys. Res. 67(8), 3151 (1962) 
[38] Wahlen, E.: Steady periodic capillary-gravity waves with vorticity. SIAM J. Math. Anal. 38, 921-943 (2006)

[39] Wahlen, E.: Steady periodic capillary waves with vorticity. Ark. Mat. 44, 367-387 (2006)

[40] Wilton, J.R.: On ripples. Phil. Mag. 29, 688-700 (1915)

[41] Yakubovich, E.I., Zenkovich, D.A.: Matrix approach to Lagrangian fluid dynamics. J. Fluid Mech. 443, 167-196 (2001)

Hung-Chu Hsu

Research Center of Ocean Environment and Technology

National Cheng Kung University

Tainan 701

Taiwan

e-mail: hchsu@thl.ncku.edu.tw

Chiu-On Ng

Department of Mechanical Engineering

The University of Hong Kong

Hong Kong

China

(accepted: October 4, 2010; published online: December 8, 2010)
Hwung-Hweng Hwung

Department of Hydraulic and Ocean Engineering

National Cheng Kung University

Tainan 701

Taiwan 